\title{
088 ASSOCIATION BETWEEN DIETARY DIVERSITY SCORE AND NUTRITIONAL STATUS OF FILIPINO CHILDREN 6-23 MONTHS
}

Mildred Ocampo Guirindola, Cristina Garcia Malabad, Lynell Valdeabella Maniego, Sheila Luz Magsino Punzalan. DOST, FNRI, Taguig, Philippines

\subsection{6/bmjopen-2015-forum2015abstracts.88}

Background Quality of diet starting at six months plays a vital role in the nutritional adequacy and nutritional status of children 6-23 months old.

Objectives This cross-sectional study aimed to test the association between quality of child' $s$ food intake as measured by dietary diversity score and nutritional status based on a one day food recall.

Methods Data of 4,276 children aged 6-23 months was taken from the country's Updating Survey conducted by the Food and Nutrition Research Institute of the Department of Science and Technology (FNRI-DOST) in June-December 2011. Child with four or more dietary diversity score was classified as meeting the minimum dietary diversity (MDD) and less than four as otherwise. Associated predictors of MDD were determined by performing bivariate analysis using Chi-square test for selected child, maternal and household characteristics and multiple regression analyses to test the association of nutritional status, MDD and other predictors.

Result The study showed that MDD was associated to underweight and wasting but not to stunting. Meeting the MDD was protective against underweight (Odds Ratio $(\mathrm{OR})=0.80$, 95\% Confidence Interval (CI) $0.64-1.00)$ and wasting $(\mathrm{OR}=0.59$, 95\% CI 0.44-0.79). Aside from not meeting the MDD, factors that increased the child' s odds to be underweight were child age at 9-11months (OR 1.83) and 12-23 months $(\mathrm{OR}=2.17)$, household food insecurity $(\mathrm{OR}=1.90)$ and mother working away from home $(\mathrm{OR}=2.42)$ while household food insecurity $(\mathrm{OR}=1.47)$ and mother working away from home (2.95) increased the probability of the child to be wasted.

Conclusion Household food insecurity, urban residence and mothers working away from home are constant predictors of underweight and wasting. Being food insecure increases the child's odds of being underweight by 1.9 times and wasting by 1.47 times while having mother who works away from home increases the odds that the child will be underweight and wasted by 2.42 times and 2.95 times, respectively. 